\title{
Thiophene Terminated Fullerene Derivatives for Interfacial Modification toward High Efficiency MAPbI 3 Perovskite Solar Cells
}

Hui Wang ${ }^{1,2 \dagger}$, Mengting Chen ${ }^{1,2 \dagger}$, Fabao Li $^{3 *}$, Rui Sun $^{3}$, Pang Wang ${ }^{1,2}$, Fanghao Ye ${ }^{1,2}$, Huijun Zhang ${ }^{1,2}$, Weiqiang Miao ${ }^{1,2}$, Dan Liu ${ }^{1,2 *}$ and Tao Wang ${ }^{1,2 *}$

${ }^{1}$ School of Materials Science and Engineering, Wuhan University of Technology, Wuhan, 430070, China E-mail: dliu@whut.edu.cn,twang@whut.edu.cn

${ }^{2}$ State Key Laboratory of Silicate Materials for Architectures, Wuhan University of Technology, Wuhan, 430070, China

${ }^{3}$ Ministry of Education Key Laboratory for the Synthesis and Application of Organic Functional Molecules, Hubei University, Wuhan 430062, China E-mail: 1fb0615@hubu.edu.cn

$\dagger$ These two authors contribute equally to this work. 


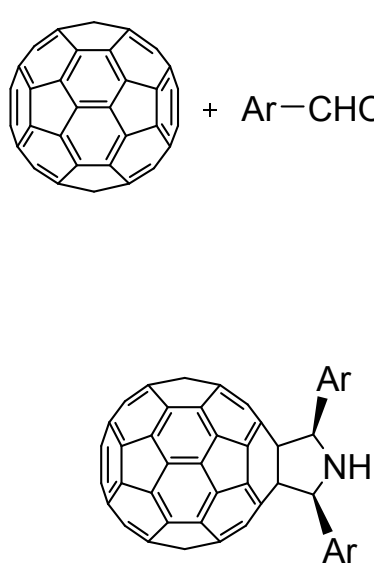

A
$+\operatorname{Ar} \widehat{\mathrm{NH}_{2}} \underset{\text { o-dichlorobenzene }}{160^{\circ} \mathrm{C}, \text { air }}$

$\mathrm{Ar}=$

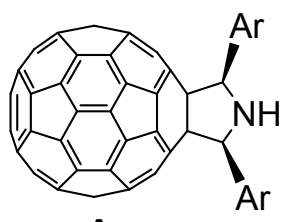

A

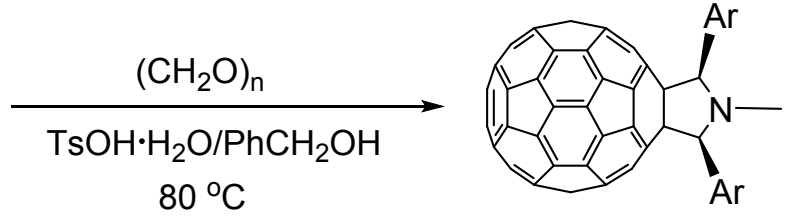

NMPPF/NMTTF

Figure S1. The synthetic routes of NMPPF and NMTTF.

Synthesis of $\boldsymbol{N}$-unsubstituted fulleropyrrolidines A: A mixture of $\mathrm{C}_{60}(0.05 \mathrm{mmol})$, aromatic aldehyde $(0.25 \mathrm{mmol})$, and arylmethylamine $(0.25 \mathrm{mmol})$ was added to a $50 \mathrm{~mL}$ round-bottom flask equipped with a reflux condenser and a magnetic stirrer. After the mixed compounds were completely dissolved in o-dichlorobenzene $(6 \mathrm{~mL})$ by sonication, the resulting solution was heated with stirring in an oil bath preset at $160{ }^{\circ} \mathrm{C}$ under air conditions. The reaction was carefully monitored by thin-layer chromatography (TLC) and stopped at the designated time. The reaction mixture was filtered through a silica gel plug in order to remove any insoluble material. After the solvent was evaporated in vacuo, the residue was separated on a silica gel column with carbon disulfide/toluene as the eluent to afford first unreacted $\mathrm{C}_{60}$, and then $N$-unsubstituted fulleropyrrolidines $\mathbf{A}$ as amorphous brown solids.

Synthesis of $\boldsymbol{N}$-methyl fulleropyrrolidines NMPPF/NMTTF: Fulleropyrrolidines A $(0.022$ $\mathrm{mmol}),\left(\mathrm{CH}_{2} \mathrm{O}\right)_{\mathrm{n}}(0.110 \mathrm{mmol}), \mathrm{TsOH} \cdot \mathrm{H}_{2} \mathrm{O}(0.044 \mathrm{mmol})$ and benzyl alcohol $(0.110 \mathrm{mmol})$ were added to a $50 \mathrm{~mL}$ round-bottom flask equipped with a reflux condenser and a magnetic stirrer. After they were completely dissolved in chlorobenzene $(6 \mathrm{~mL})$ by sonication, the resulting solution was put into an oil bath preset at $80{ }^{\circ} \mathrm{C}$ and stirred under air conditions. Thin-layer chromatography (TLC) was employed to carefully monitor the reaction and to stop the reaction at the designated time. The reaction mixture was filtered through a silica gel plug to remove any insoluble material. After the solvent evaporation in vacuo was completed, the residue was separated on a silica gel column with $\mathrm{CS}_{2}$ as the eluent to afforded first NMPPF/NMTTF as amorphous brown solid (mp > $300^{\circ} \mathrm{C}$ ) and then unreacted $\mathbf{A}$. 
NMPPF: ${ }^{1} \mathrm{H}$ NMR $\left(500 \mathrm{MHz}, \mathrm{CS}_{2} / \mathrm{DMSO}_{6}\right) \delta 8.14(\mathrm{~d}, J=8.2 \mathrm{~Hz}, 2 \mathrm{H}), 7.58-7.56(\mathrm{~m}, 2 \mathrm{H})$, 7.47-7.44 (m, 2H), 7.33-7.27 (m, 4H), $5.16(\mathrm{~s}, 2 \mathrm{H}), 2.63(\mathrm{~s}, 3 \mathrm{H}) ;{ }^{13} \mathrm{C} \mathrm{NMR}(125 \mathrm{MHz}$, $\mathrm{CS}_{2} / \mathrm{DMSO}_{-}{ }_{6}$ ) (all $2 \mathrm{C}$ unless indicated) $\delta 152.89,152.61,146.24,145.73,145.24,145.17,145.06$, 144.98, 144.82, 144.67 (1C), 144.54, 144.52 (1C), 144.23, 144.14, 144.07, 143.62, 143.35, 142.10 (1C), 141.96 (1C), 141.60, 141.51, 141.17, 141.12, 141.02, 140.94, 140.84, 140.49, 138.95, 138.34, 135.97, 135.76, 134.73, 129.24 (aryl C), 128.52 (aryl C), 127.75 (aryl C), 127.68 (aryl C), 127.56 (aryl C), 82.34, 74.37, 38.10 (1C); FT-IR v/cm ${ }^{-1}$ (KBr) 1603, 1495, 1453, 1428, 1279, 1182, 1026, 706, 698, 574, 526; UV-vis $\left(\mathrm{CHCl}_{3}\right) \lambda_{\max } / \mathrm{nm} 258,310,431 ; \mathrm{HRMS}$ (MALDI-TOF) m/z: [M] ${ }^{-}$ Calcd for $\mathrm{C}_{75} \mathrm{H}_{15} \mathrm{~N}$ 929.1205; Found 929.1199.

NMTTF: ${ }^{1} \mathrm{H}$ NMR (500 MHz, $\left.\mathrm{CS}_{2} / \mathrm{DMSO}_{6}\right) \delta 7.39$ (br.s, 2H), 7.37 (d, $\left.J=5.2 \mathrm{~Hz}, 2 \mathrm{H}\right), 7.00$ (t, $J$ $=4.2 \mathrm{~Hz}, 2 \mathrm{H}), 5.51(\mathrm{~s}, 2 \mathrm{H}), 2.78(\mathrm{~s}, 3 \mathrm{H}) ;{ }^{13} \mathrm{C} \mathrm{NMR}\left(125 \mathrm{MHz}, \mathrm{CS}_{2} / \mathrm{DMSO}-d_{6}\right)$ (all 2C unless indicated) $\delta 151.70,151.35,145.39,144.86,144.40,144.28,144.16$ (4C), 143.95, 143.83 (1C), 143.72, 143.59 (1C), 143.37, 143.31, 143.20, 142.77, 142.43, 141.23 (1C), 141.04 (1C), 140.73, 140.67, 140.27, 140.19, 140.15, 140.02 (4C), 139.64, 138.73 (aryl C), 138.03, 137.55, 135.16, 133.79, $126.43(\operatorname{aryl} C), 126.01(\operatorname{aryl} C), 125.39$ (aryl C), 77.04, 73.15, 38.09 (1C); FT-IR v/cm ${ }^{-1}$ (KBr) 1537, 1456, 1431, 1298, 1234, 1181, 1033, 833, 776, 701, 670, 570, 528; UV-vis $\left(\mathrm{CHCl}_{3}\right)$ $\lambda_{\max } / \mathrm{nm}$ 257, 311, 431; HRMS (MALDI-TOF) m/z: [M] ${ }^{-}$Calcd for $\mathrm{C}_{71} \mathrm{H}_{11} \mathrm{NS}_{2}$ 941.0333; Found 941.0331 . 
(a)

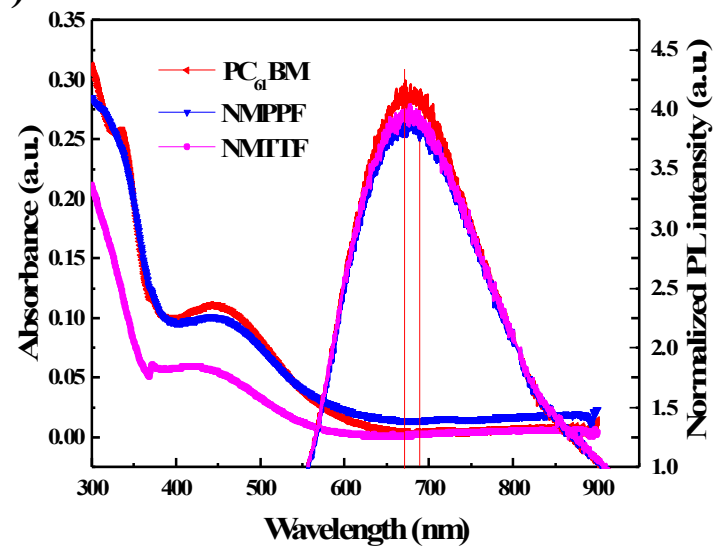

(c)

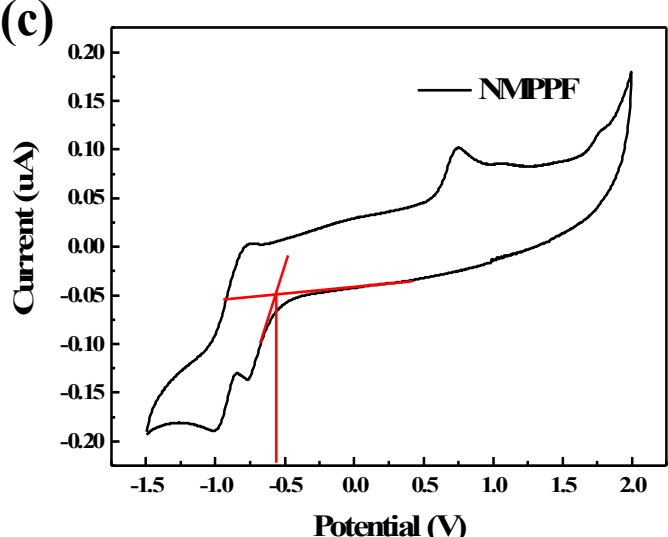

(e)

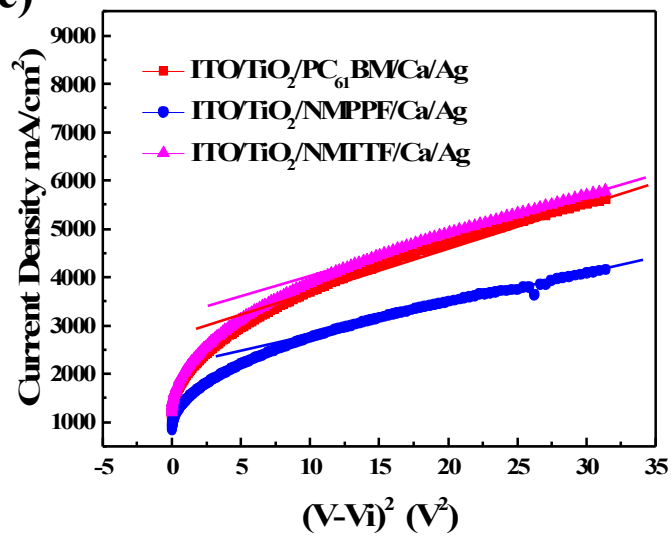

(b)

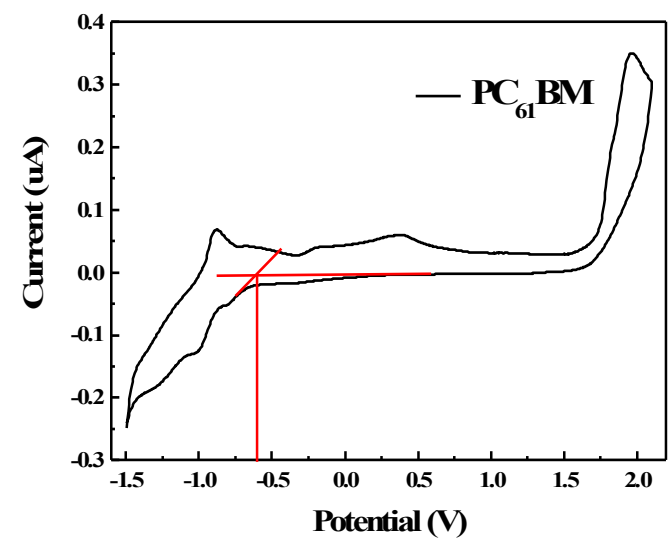

(d)

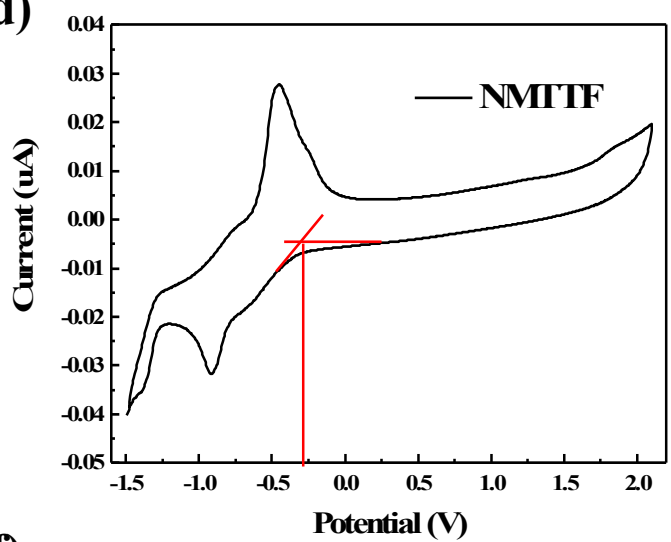

(f)

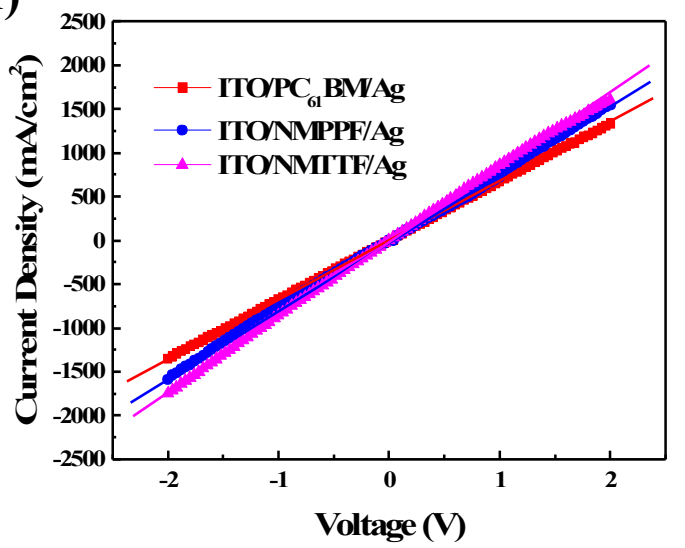

Figure S2. (a) PL and UV-Vis spectra of fullerene derivatives. Cyclic voltammograms of (b) $\mathrm{PC}_{61} \mathrm{BM}$, (c) NMPPF and (d) NMTTF. The dark J-V curves to calculate (e) mobility and (f) conductivity of fullerene derivatives. 


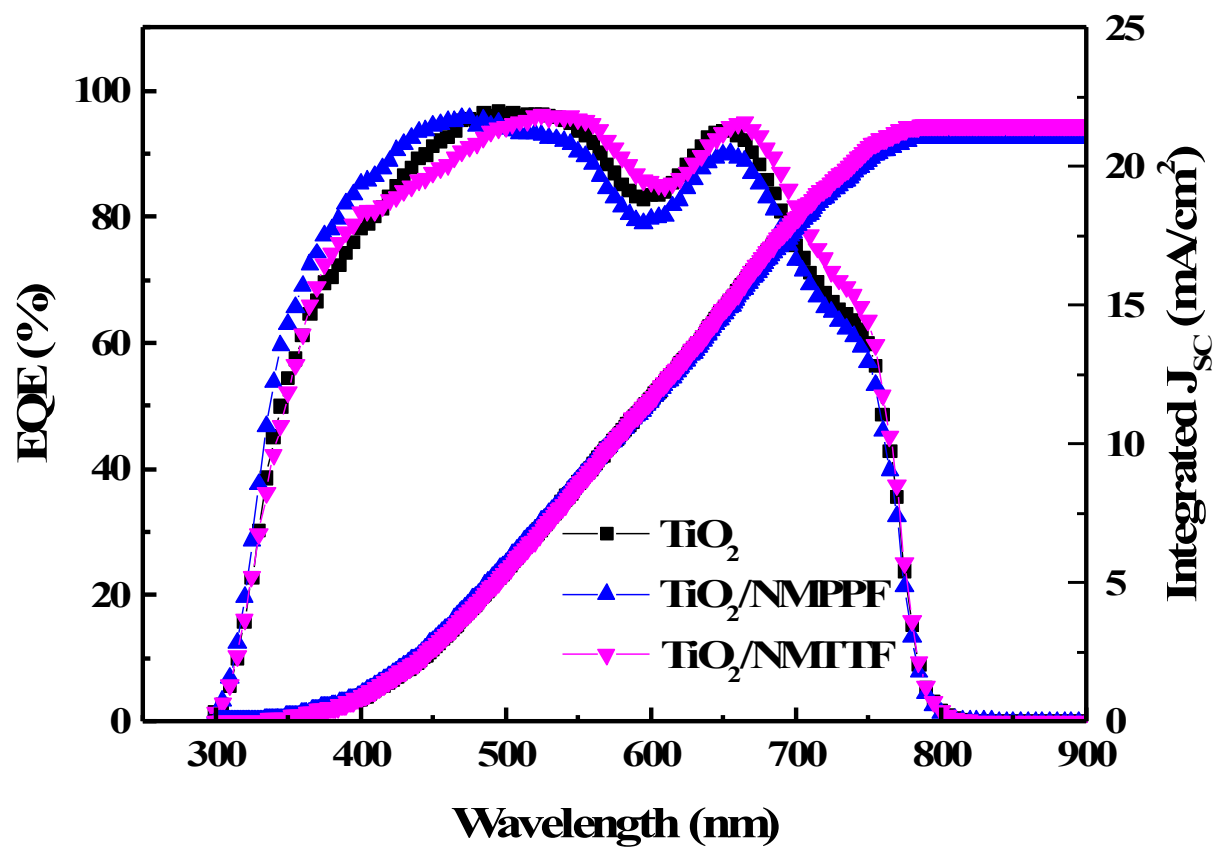

Figure S3. External quantum efficiency of PSCs without and with an NMPPF or NMTTF interlayer.
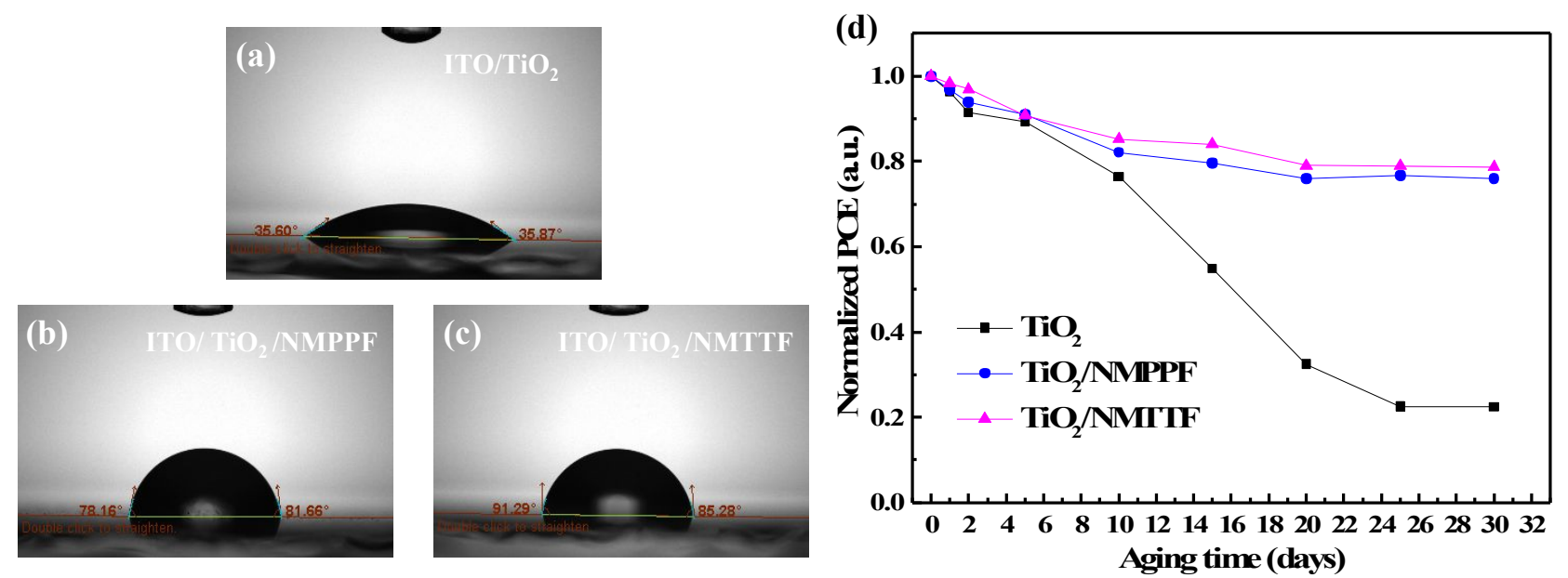

Figure S4. (a-d) Water contact angle measurements on different fullerene derivatives. (e) Evolution of device PCE obtained by storing unencapsulated PSCs in ambient condition with a relative humidity of $40 \pm 5 \%$ and an average temperature of $25^{\circ} \mathrm{C}$. 
(a)

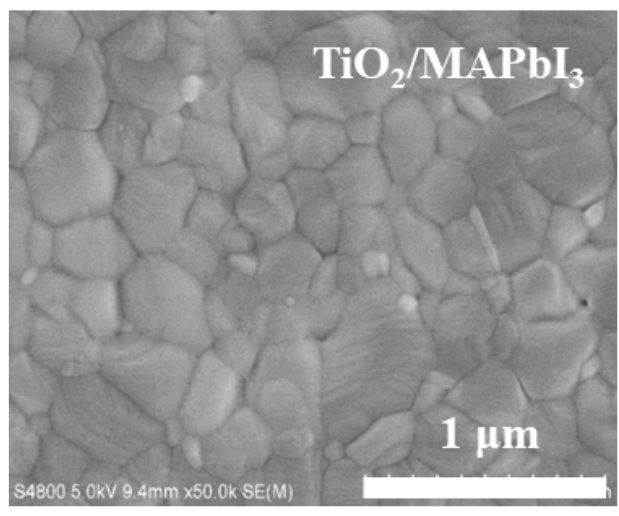

(c)

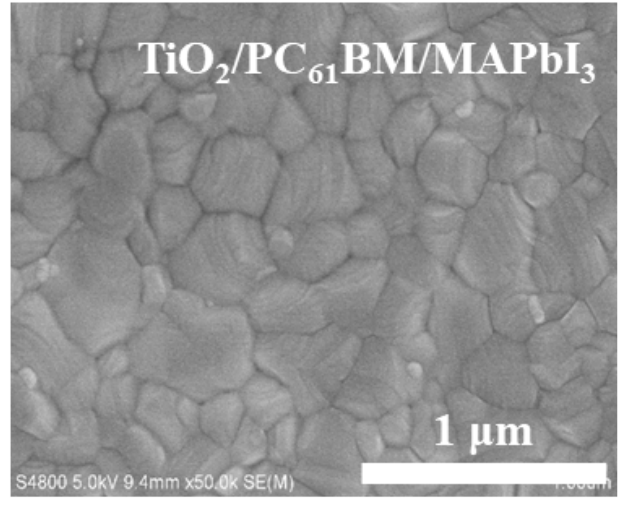

(e)

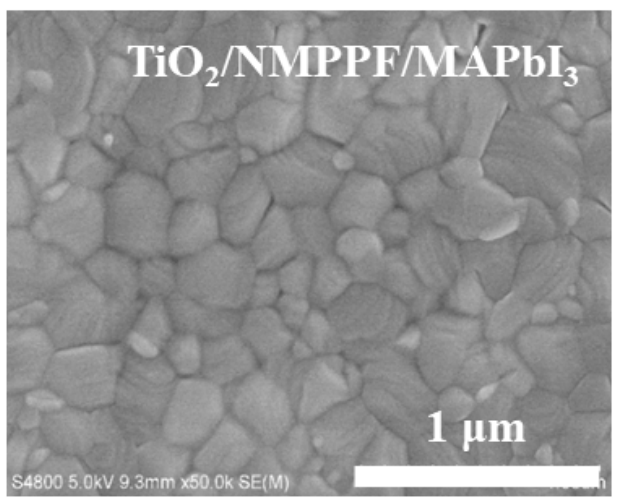

(g)

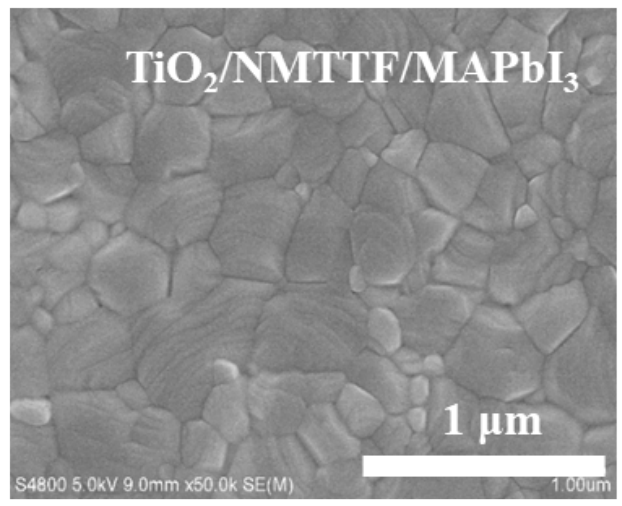

(b)

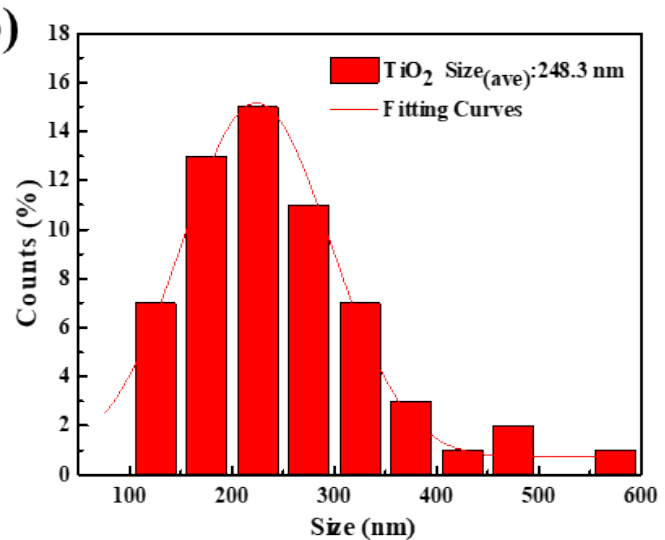

(d)

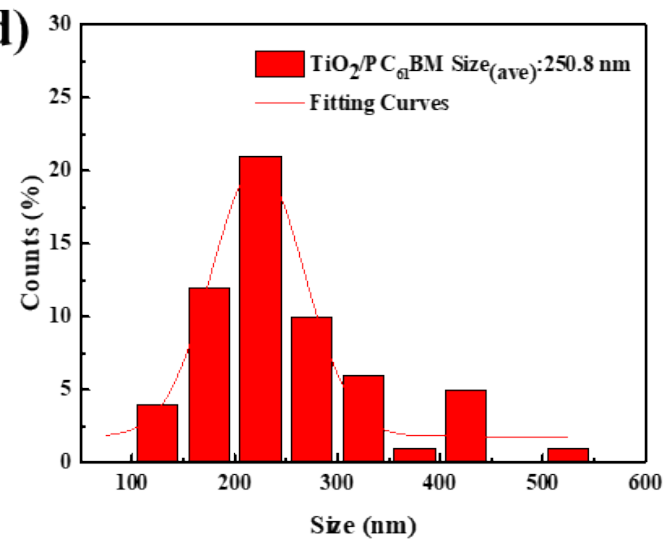

(f)

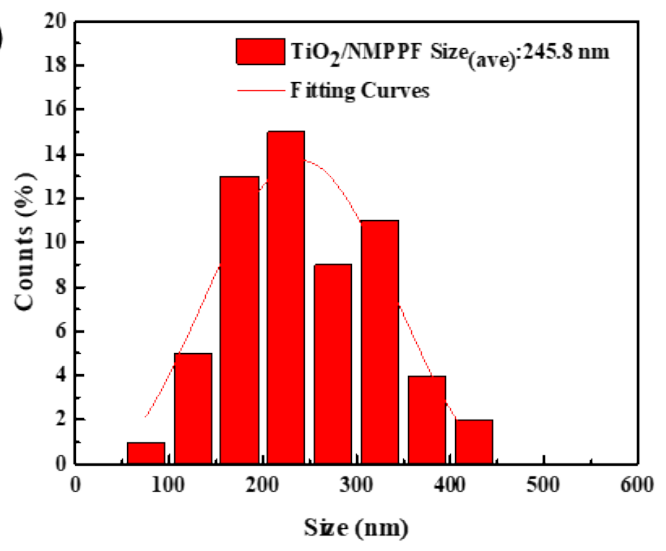

(h)

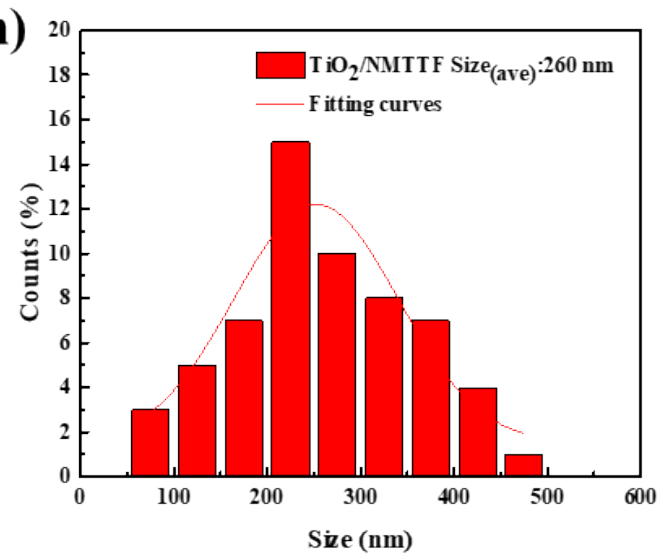

Figure S5. SEM images of perovskite films cast on different fullerene derivative surfaces: (a) pure $\mathrm{TiO}_{2}$, (b) $\mathrm{TiO}_{2} / \mathrm{NMPPF}$, (c) $\mathrm{TiO}_{2} / \mathrm{NMTTF}$, (d) $\mathrm{TiO}_{2} /$ NMTTF-Cl. 

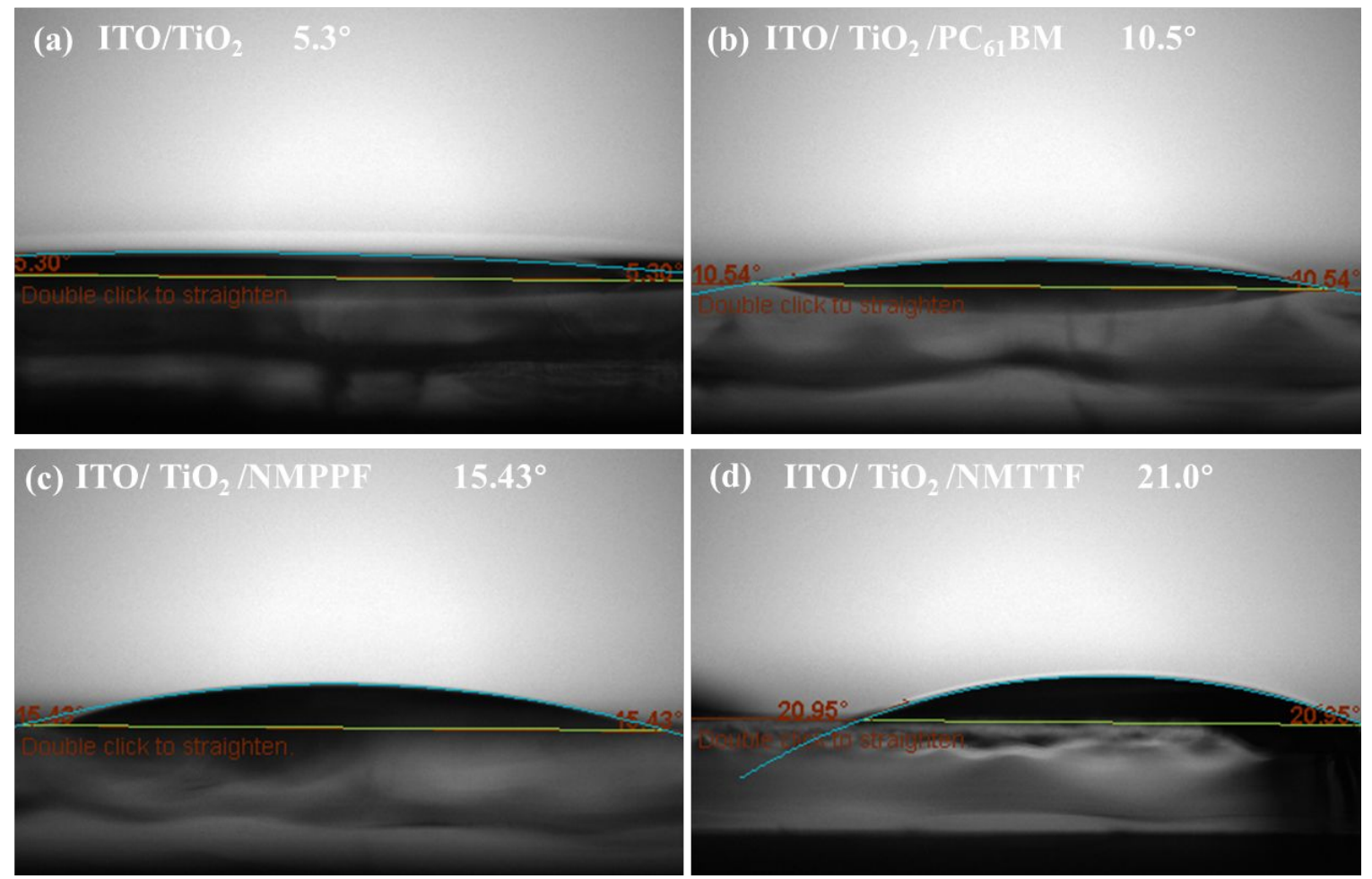

Figure S6. (a-d) Contact angle of perovskite precursor on different fullerene film surfaces.

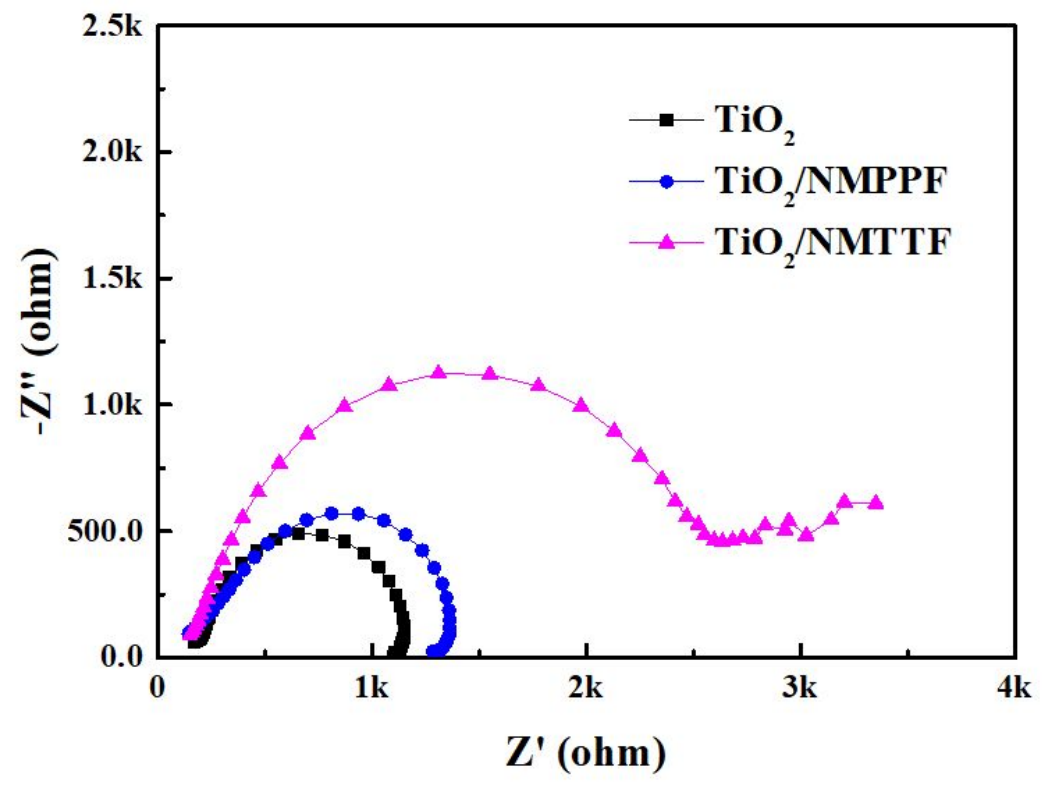

Figure S7. Nyquist plots for PSCs with and without fullerene interlayers at an applied bias of $0.6 \mathrm{~V}$ 